\title{
Teoria da Mente em Pais de Pessoas com Autismo: Uma Análise Comparativa
}

\section{Theory of Mind for Parents of People with Autism: A Comparative Analysis}

\author{
Aline Abreu e Andrade*, a, Walter Camargos Junior ${ }^{\mathrm{b}}$, Priscilla Moreira Ohno ${ }^{\mathrm{a}}$ \\ \& Maycoln Leôni Martins Teodoro ${ }^{\mathrm{a}}$ \\ ${ }^{a}$ Universidade Federal de Minas Gerais, Belo Horizonte, MG, Brasil \\ $\&{ }^{b}$ Hospital Infantil João Paulo II, Belo Horizonte, MG, Brasil
}

\begin{abstract}
Resumo
O autismo pode ser definido como uma síndrome comportamental heterogênea em termos genéticos e de diagnóstico. Esta heterogeneidade dificulta a realização de estudos genéticos sobre o tema. Um dos endofenótipos candidatos do autismo é a teoria da mente. O objetivo deste estudo foi avaliar a capacidade da teoria da mente em pais de crianças com autismo. Foram avaliados 90 participantes: 30 pais de crianças com autismo, 30 pais de crianças com síndrome de Down e 30 pais de crianças com desenvolvimento típico. Os instrumentos utilizados foram o Eyes Test, para avaliar habilidades de decodificação, e o Unexpected Outcomes Task, para medir o raciocínio dedutivo. Os resultados não indicam diferenças significativas entre os grupos na habilidade de decodificação. No entanto, eles indicam a existência de déficits na capacidade de dedução da teoria da mente. Esse déficit parece ser mais pronunciado em mães de crianças com autismo, em comparação com grupos de mães de crianças com síndrome de Down e mães de crianças com desenvolvimento típico. Mais estudos são necessários para esclarecer melhor a relação entre teoria da mente e autismo.

Palavras-chave: Teoria da mente, autismo, avaliação, pais.
\end{abstract}

\begin{abstract}
Autism can be defined as a heterogeneous behavioral syndrome in both genetic and diagnostic terms. This heterogeneity poses difficulties in conducting genetic studies on the topic. A candidate endophenotype of autism is theory of mind. The goal of this study was to evaluate the ability of theory of mind in parents of children with autism. We evaluated 90 participants: 30 parents of children with autism, 30 parents of children with Down syndrome and 30 parents of typically developing children. The instruments used were the Eyes Test, to assess decoding skills, and the Unexpected Outcomes Task, to measure deductive reasoning. The results do not indicate significant differences between groups in theory of mind decoding. However, they indicate the existence of deficits in the theory of mind reasoning. These deficits seem to be more pronounced in mothers of children with autism, compared to groups of mothers of children with Down syndrome and mothers of typically developing children. Further studies are needed to better clarify the relationship between theory of mind and autism.

Keywords: Theory of mind, autism, assessment, parents.
\end{abstract}

O autismo é uma síndrome comportamental que envolve a presença de prejuízos nas habilidades de interação social e comunicação, bem como de padrões restritos e repetitivos de comportamento, interesses e atividades. $\mathrm{O}$ transtorno se manifesta precocemente no desenvolvimento e possui taxa de prevalência de $0,5 \%$ da população (Associação Americana de Psiquiatria, 2014; Fombonne, 2009).

Do ponto de vista genético, o autismo é considerado um transtorno heterogêneo, na medida em que envolve a interação de múltiplos genes, que juntos levam à pre-

" Endereço para correspondência: Rua Aimorés, 2710,

Bairro Lourdes, Belo Horizonte, MG, Brasil 30140-072.

E-mail: aline abreu andrade@yahoo.com.br disposição à manifestação do transtorno em sua forma completa (Betancur, 2011). Desde o estudo de Folstein e Rutter (1977), no qual as bases genéticas do autismo foram demonstradas de forma convincente pela primeira vez, evidências vêm sendo acumuladas neste sentido. Apesar disto, ainda não estão claros quais genes estão implicados no transtorno (Lichtenstein, Carlstrom, Rastam, Gillberg, \& Anckarsater, 2010).

Em termos diagnósticos, há uma tendência atual na literatura em definir o autismo através de uma abordagem dimensional, sendo o transtorno autista considerado como o extremo de um continuum de traços autísticos (Scheeren \& Stauder, 2008). Esta noção de transtorno espectral remete à ideia de que os indivíduos podem ser afetados em 
diferentes graus (Constantino, 2011). Questiona-se, assim, a existência de várias entidades psiquiátricas distintas em favor de um conceito único, denominado transtornos do espectro do autismo (TEA).

Vários estudos demonstram que familiares de indivíduos com TEA exibem traços subclínicos relacionados ao transtorno, tais como déficits sociais e de linguagem (Kanne, Christ, \& Reiersen, 2009; Mecca et al., 2011). Embora geralmente muito sutis em sua expressão, essas características se relacionam intimamente aos principais domínios de sintomas do autismo (ou seja, interesses restritos e repetitivos, comprometimento social e déficits de comunicação), e têm sido denominados coletivamente como o fenótipo ampliado do autismo (FAA).

Fenótipo ampliado do autismo se refere à manifestação de traços autísticos subclínicos em familiares de pessoas com autismo, que são insuficientes para se constituir em um diagnóstico. Estudos que indicam expressões brandas de aspectos relacionados ao autismo em familiares podem ser interpretados em termos de uma possível suscetibilidade genética para o transtorno completo. As evidências sobre a alta concordância de características autísticas subsindrômicas entre irmãos, por exemplo, são um indicativo de vulnerabilidade genética para as variações mais brandas do autismo (Scheeren \& Stauder, 2008).

Atualmente, além dos estudos genéticos, tem-se utilizado abordagens adicionais para a identificação de genes de suscetibilidade (Jonas \& Markon, 2014). Uma dessas estratégias se refere ao estudo de fenótipos pré-comportamentais qualitativamente semelhantes às características do autismo, porém mais brandos, presentes entre os familiares de indivíduos autistas, que parecem se constituir em uma expressão geneticamente significativa de algumas características componentes do autismo (Losh \& Piven, 2007). Estas medidas neurocognitivas podem ser uma ferramenta poderosa para o avanço do conhecimento sobre a vulnerabilidade genética para o autismo (Rommelse, Geurts, Franke, Buitelaar, \& Hartman, 2011). A estes fenótipos dá-se o nome de endofenótipos.

Os endofenótipos se constituem em fatores de risco que vão além do comportamento observável e podem ser investigados a partir de medidas bioquímicas, neurofisiológicas, neuroanatômicas e, neuropsicológicas (Hu et al., 2011). Eles possuem uma maior correlação com os fatores genéticos do que com os comportamentos característicos da síndrome. O estudo dos endofenótipos tem surgido, assim, como um campo atual de estudo, no sentido de viabilizar o esclarecimento das relações gene-cérebro-comportamento (Gottesman \& Gould, 2003).

A identificação de endofenótipos nos TEA pode fornecer um meio para precisar mecanismos neuropsicológicos de importância biológica e genética para o transtorno (Losh \& Piven, 2007). Um dos mais promissores candidatos a endofenótipo do autismo é a disfunção neurocognitiva (Happé \& Ronald, 2010). Há três supostos endofenótipos cognitivos que vêm sendo estudados para o autismo: déficits de teoria da mente, função executiva e fraca coerência central. Tais hipóteses cognitivas do autismo se propõem a lançar luz a diferentes características centrais ao transtorno (Best, Moffat, Power, Owens, \& Johnstone, 2008; Pellicano, Maybery, Durkin, \& Maley 2006).

Os déficits de teoria da mente (ToM) vêm sendo apontados em estudos de neurocognição como um dos possíveis marcadores endofenotípicos de TEA (Nýden, Hagberg, Goussé, \& Rastam, 2011). O conceito de teoria da mente surgiu no fim da década de 70 a partir de experimentos sobre cognição animal e é definido como a capacidade de atribuir estados mentais para si e para os outros, desenvolvendo uma concepção daquilo que eles pensam, sentem, desejam, acreditam e duvidam (Premack \& Woodruff, 1978).

Tager-Flusberg e Sullivan (2000) propuseram que a ToM incluiria componentes sociocognitivos e socioperceptuais. A partir dessa visão componencial, foi desenvolvida uma perspectiva mais funcional, em que ToM é separada em um processo de duas fases: decodificação e dedução do estado mental. O primeiro se refere à capacidade de identificar os estados mentais de outra pessoa com base em informações observáveis, como expressões faciais, gestos ou tom de voz. Já o segundo componente envolve a capacidade de integrar as informações contextuais e históricas sobre uma pessoa (suas atitudes, conhecimentos e experiências) para entender e predizer o seu comportamento. Na fase inicial, sinais relevantes são decodificados. No segundo estágio, a informação decodificada é usada para interpretar os estados mentais (Njomboro, Deb, \& Humphreys, 2008; Sabbagh, 2004).

Há crescentes evidências de que os dois componentes da teoria da mente se relacionam a circuitos cerebrais diferentes e não se sobrepõem (Caoa, Lib, Lia, \& Lia, 2012; Njomboro et al., 2008; Sabbagh, 2004; Tager-Flusberg \& Sullivan, 2000). A decodificação do estado mental seria um estágio inicial, enquanto a habilidade de dedução do estado mental seria a segunda etapa do processo (Njomboro et al., 2008; Sabbagh, 2004). A existência de diferentes redes neurais associadas aos componentes da ToM aponta para a possibilidade de que eles sejam investigados separadamente (Sabbagh, 2004).

Sabe-se que crianças com autismo apresentam resultado abaixo do esperado em tarefas que avaliam teoria da mente, tanto no que se refere à decodificação, quanto à dedução de estados mentais. Estes déficits já foram amplamente documentados na literatura (Best et al., 2008; Pellicano et al., 2006). Considerando-se as evidências de que a herdabilidade no autismo se encontra em torno de $80 \%$ (Ronald \& Hoekstra, 2011), hipotetiza-se sobre a presença de déficit de teoria da mente também em familiares de pessoas com autismo.

Mas embora déficits de ToM tenham sido amplamente estudados em pacientes autistas, ainda não foram suficientemente investigados em seus familiares (Viding \& Blakemore, 2007). Em pesquisa com este enfoque, Gokcen, Bora, Erermis, Kesikci e Aydin (2009) pesquisaram famílias com crianças autistas e demonstraram 
Andrade, A. A., Camargos Junior, W., Ohno, P. M. \& Teodoro, M. L. M. (2015). Teoria da Mente em Pais de Pessoas com Autismo: Uma Análise Comparativa.

que adultos não portadores de autismo, mas que possuem filhos com autismo apresentam déficit de teoria da mente, com especial comprometimento na dedução a respeito das emoções de outras pessoas. Uma vez que a disfunção na interação social está dentre as características centrais do autismo, a teoria da mente e, mais especificamente, a habilidade de dedução surge como candidata a marcador endofenotípico do autismo.

O estudo realizado por Gokcen et al. (2009) provê evidência a favor desta possibilidade, podendo trazer subsídio à compreensão da síndrome. $\mathrm{O}$ objetivo da presente pesquisa consiste em dar continuidade à investigação dessa hipótese, avaliando separadamente pais e mães no que se refere à habilidade de teoria da mente e incluindo no estudo uma amostra de pais de crianças com Síndrome de Down, de modo a avaliar se a presença de uma doença crônica na família, por si só, afeta os resultados de ToM dos pais. Esta comparação permite avaliar se os déficits de ToM se relacionam especificamente ao transtorno autista, ou se são devidos ao contexto estressor ao qual os pais de doenças crônicas estão submetidos (Andrade \& Teodoro, 2012). Hipotetiza-se, assim, que os pais de crianças com autismo apresentem déficit de teoria da mente em relação aos dois outros grupos, enquanto que o grupo de pais de crianças com Síndrome de Down não apresente diferenças significativas em relação ao grupo típico.

\section{Método}

\section{Amostra}

Os participantes foram recrutados no Ambulatório de Transtornos Complexos do Desenvolvimento Infantil do Hospital Infantil João Paulo II (HIJP-II), instituição pública vinculada à Fundação Hospitalar do Estado de Minas Gerais, especializada em assistência pediátrica.

Participaram da pesquisa três grupos de pais (Tabela 1). O Grupo I foi composto por 15 pais e 15 mães biológicas de crianças com diagnóstico de transtorno autista independente do grau de comprometimento do transtorno. O diagnóstico de transtorno autista no Grupo I foi firmado por psiquiatra sênior utilizando-se os critérios do Manual Diagnóstico e Estatístico dos Transtornos Mentais (DSM-IV-TR; Associação Americana de Psiquiatria, 2002) e do Childhood Autism Rating Scale (CARS).

O Grupo II foi pareado com o Grupo I com relação ao sexo e escolaridade e foi constituído por 10 pais e 20 mães de crianças com síndrome de Down. O Grupo III, também pareado com o Grupo I em relação ao sexo e escolaridade, foi constituído por 15 pais e 15 mães de crianças com desenvolvimento típico, de modo a mensurar a diferença entre ausência e presença de um transtorno crônico na criança. Assim, foram incluídos pais cujos filhos não apresentassem qualquer transtorno psiquiátrico, avaliação realizada por meio de entrevista e da Escala de Comportamentos da Infância e Adolescência (Bordin, Mari, \& Caeiro, 1995) / The Child Behavior Checklist (CBCL; Achenbach \& Edelbrock, 1983), que busca a detecção de problemas comportamentais ou desenvolvimentais nos filhos dos possíveis participantes da pesquisa, funcionando como critério de exclusão para o Grupo III.

Em todos os grupos, foram definidos como critérios de exclusão pais que obtivessem escores de inteligência inferiores a dois desvio-padrão abaixo da média e pais com histórico pessoal ou familiar (de primeiro grau) de transtornos psiquiátricos. No Grupo I foram critérios de exclusão pais de crianças com autismo (a) com história pregressa de nascimento com menos de 35 semanas e/ou abaixo de $2,5 \mathrm{Kg}$ e; (b) com história pregressa de quadros neurológicos que causassem comprometimentos no desenvolvimento infantil. Para o Grupo II convencionou-se que não participariam da pesquisa pais de crianças com síndrome de Down em comorbidade com autismo e aqueles que possuíam um filho com autismo. No Grupo III foram excluídos pais de crianças que apresentassem alguma sintomatologia ou diagnóstico psiquiátrico. Com base nestes critérios de seleção da amostra houve a exclusão de 14 participantes da pesquisa.

Tabela 1

Média e Desvio-Padrão da Idade e Escores no Wechsler Adult Intelligence Scale-Revised (WAIS-R) dos Participantes

\begin{tabular}{|c|c|c|c|c|c|c|}
\hline & \multicolumn{2}{|c|}{$\begin{array}{c}\text { Grupo I - Pais de Crianças com } \\
\text { autismo }\end{array}$} & \multicolumn{2}{|c|}{$\begin{array}{c}\text { Grupo II - Pais de Crianças } \\
\text { com Down }\end{array}$} & \multicolumn{2}{|c|}{$\begin{array}{c}\text { Grupo III - Pais de Crianças } \\
\text { típicas }\end{array}$} \\
\hline & Pai $(n=15)$ & Mãe $(n=15)$ & Pai $(n=10)$ & Mãe $(n=20)$ & Pai $(n=15)$ & Mãe $(n=15)$ \\
\hline Idade (Média, $D P$ ) & $35,79(5,62)$ & $35,60(5,63)$ & $41,30(4,72)$ & $41,35(7,73)$ & $36,07(5,15)$ & $34,73(5,20)$ \\
\hline Wais-R (Média, DP) & $78,60(26,40)$ & $79,67(24,57)$ & $100,80(16,69)$ & $88,15(18,13)$ & $86,93(27,25)$ & $88,67(25,37)$ \\
\hline \multicolumn{7}{|c|}{ Escolaridade (Frequência, \%) } \\
\hline Ensino fundamental & $2(13,3 \%)$ & $2(13,3 \%)$ & $3(30,0 \%)$ & $2(9,5 \%)$ & $1(6,7 \%)$ & $3(20,0 \%)$ \\
\hline Segundo grau & $3(20,0 \%)$ & $8(53,3 \%)$ & $4(40,0 \%)$ & $5(23,8 \%)$ & $4(26,7 \%)$ & $5(33,3 \%)$ \\
\hline Curso superior & $8(53,3 \%)$ & $4(26,7 \%)$ & $3(30,0 \%)$ & $11(52,4 \%)$ & $6(40,0 \%)$ & $4(26,7 \%)$ \\
\hline Pós graduação & $2(13,3 \%)$ & $1(6,7 \%)$ & $0 \%$ & $2(9,5 \%)$ & $4(26,7 \%)$ & $3(20,0 \%)$ \\
\hline
\end{tabular}


Foram avaliados ao todo 90 pais (Tabela 1). A avaliação foi realizada predominantemente com casais. A exceção a isto se deveu à dificuldade de acesso à amostra de pais (do sexo masculino) de crianças com síndrome de Down, já documentada na literatura (Losh \& Piven, 2007). Por este motivo o Grupo I e o Grupo III foram constituídos por 15 casais, enquanto o Grupo II possuía 10 casais e 10 mães.

Os grupos foram pareados por escolaridade e inteligência através do teste qui-quadrado, não havendo discrepâncias em relação a estas variáveis. No que se refere, especificamente, aos escores brutos de pontuação nos quatro subtestes do WAIS-R administrados aos participantes, os grupos também não apresentaram diferenças significativas.

\section{Instrumentos}

Foi utilizada na triagem dos participantes uma entrevista constando os critérios de inclusão/exclusão. Foram aplicados quatro subtestes do WAIS-R (Nascimento, 2004; Wechsler, 1981) para avaliar o nível de inteligência dos pais (subtestes cubos, compreensão, semelhanças e completar figuras), conforme a escolha realizada na pesquisa de Gokcen et al. (2009).

Além disso, foi realizada a aplicação das seguintes tarefas de teoria da mente: Eyes Test e Unexpected Outcomes Task. O Eyes Test (Baron-Cohen, Jolliffe, Mortimore, \& Robertson, 1997) é constituído por 36 fotos de expressões faciais, mostrando apenas a região dos olhos. O examinando deve escolher qual, dentre quatro emoções, corresponde à expressão dos olhos apresentada na foto. Com isto, o instrumento objetiva avaliar a habilidade de decodificação da ToM. Estudo recente sobre o Eyes Test, investigando amostras com idade entre 18 e 32 anos, indicou consistência interna adequada (superior a 0,60 ) e boa estabilidade teste-reteste ( $r=0,83$; Vellante et al., 2012). Além disso, o instrumento apresenta resultados discriminantes entre amostras clínicas e controles (Baron-Cohen et al., 1997).

O Unexpected Outcomes Task(UOT; Dyck, Ferguson, $\&$ Shochet, 2001) possui 12 histórias nas quais existem aparentes incongruências de emoção. O examinando deve identificar possíveis causas da emoção incongruente presente na narrativa. Com isto, o UOT visa avaliar a habilidade de dedução de ToM. O instrumento apresentou consistência interna de 0,82 em estudo piloto realizado por Dyck et al. (2001) com adolescentes e adultos. A correção do teste foi realizada segundo os critérios expostos no Gabarito.

A avaliação foi realizada em uma sala do Hospital Infantil João Paulo II e o tempo de aplicação dos instrumentos foi de em média uma hora e trinta minutos. As entrevistas foram conduzidas pela autora principal do artigo e por duas alunas de iniciação científica.

\section{Procedimentos}

A pesquisa foi aprovada pelo Comitê de Ética em Pesquisa da Fundação Hospitalar de Minas Gerais (FHEMIG). $\mathrm{O}$ recrutamento de participantes foi realizado no Ambulatório de Transtornos Complexos do Desenvolvimento Infantil do HIJP-II. Os pais foram informados sobre a natureza do estudo e o seu consentimento foi documentado por meio do Termo de Consentimento Livre e Esclarecido. Após o consentimento foi realizada a coleta dos dados.

\section{Análises}

As comparações entre os três grupos foram realizadas com Análises de Variância One-way (ANOVA) com post hoc de Bonferroni, uma vez que a amostra apresenta distribuição normal e que análises preliminares com testes não-paramétricos apontaram os mesmos resultados. Para as análises de correlações foram utilizadas Correlações de Pearson. O nível de significância utilizado foi de $p<0,05$.

\section{Resultados}

A habilidade de decodificação da teoria da mente, avaliada por meio do Eyes Test, não apresentou diferença significativa entre os três grupos. Avaliando-se separadamente as mães, também não houve discrepância entre os resultados do grupo materno de crianças com transtorno autista (Grupo I), com síndrome de Down (Grupo II) e com desenvolvimento típico (Grupo III) na avaliação da habilidade de decodificação de ToM. A avaliação também não indicou diferença significativa na pontuação dos pais de crianças com autismo, com síndrome de Down e com desenvolvimento típico (Tabela 2).

Já a habilidade de dedução da teoria da mente, investigada através do Unexpected Outcomes Task, apresentou diferenças significativas entre os três grupos avaliados, com os pais de crianças com autismo demonstrando pontuações inferiores aos pais de crianças com síndrome de Down e de crianças com desenvolvimento típico (Tabela 2).

Tabela 2

Média e Desvio-Padrão dos Resultados dos Instrumentos de ToM por Grupo $(n=90)$

\begin{tabular}{lccccccc}
\hline & \multicolumn{2}{c}{ Grupo I } & \multicolumn{2}{c}{ Grupo II } & \multicolumn{2}{c}{ Grupo III } & $F$, sig. \\
\cline { 2 - 8 } & Média & $D P$ & Média & $D P$ & Média & $D P$ & \\
\hline Eyes Test & 21,43 & 3,56 & 20,90 & 3,76 & 20,57 & 3,62 & 0,43 \\
UOT & 11,67 & 3,73 & 14,00 & 2,96 & 13,70 & 2,76 & $4,80^{*, \mathrm{a}, \mathrm{b}}$ \\
\hline
\end{tabular}

Nota. ${ }^{*} F=0,05$.

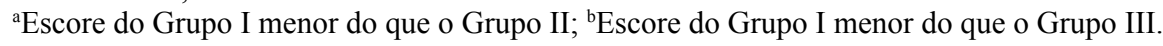


Andrade, A. A., Camargos Junior, W., Ohno, P. M. \& Teodoro, M. L. M. (2015). Teoria da Mente em Pais de Pessoas com Autismo: Uma Análise Comparativa.

$\mathrm{Na}$ investigação sobre a correlação entre inteligência e teoria da mente, os resultados dos pais em ToM foram associados à sua pontuação no WAIS-R. Estas associações foram demonstradas tanto para a habilidade de decodificação de ToM $(r=0,30, p<0,01)$ quanto na capacidade de dedução $(r=0,36, p<0,01)$.

Quando avaliados separadamente, pais e mães apresentaram resultados distintos com relação às habilidades de decodificação e dedução. Como pode ser observado na Tabela 3, não houve diferença significativa com relação à decodificação entre os grupos de mães. Por outro lado, a capacidade de dedução diferiu entre os grupos de mães. As mães de crianças com autismo apresentaram resultados significativamente inferiores às mães de crianças com Síndrome de Down e às mães de crianças típicas. Não houve diferença entre os grupos de mães de Síndrome de Down e crianças típicas.

Com relação aos pais, não houve diferença significativa entre os grupos nas pontuações dos instrumentos de teoria da mente. Os resultados estão descritos na Tabela 4.

Tabela 3

Média e Desvio-Padrão dos Resultados das Mães nos Instrumentos de ToM (n=50)

\begin{tabular}{lcccc}
\hline & Grupo I $(n=15)$ & Grupo II $(n=20)$ & Grupo III $(n=15)$ & $F$, Sig. \\
\hline Eyes Test & $22,13(3,14)$ & $20,65(3,53)$ & $21,73(2,82)$ & 1,02 \\
UOT & $11,0(3,46)$ & $13,70(2,99)$ & $14,93(2,49)$ & $6,77^{*}, \mathrm{a}, \mathrm{b}$ \\
\hline
\end{tabular}

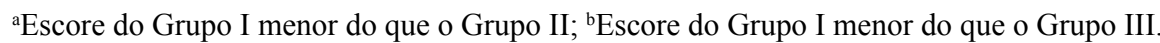
$* p<0,05$.

Tabela 4

Média e Desvio-Padrão dos Resultados dos Pais nos Instrumentos de ToM $(\mathrm{n}=40)$

\begin{tabular}{lcccc}
\hline & Grupo I $(n=15)$ & Grupo II $(n=10)$ & Grupo III $(n=15)$ & $F$, Sig. \\
\hline Eyes Test & $20,73(3,92)$ & $21,40(4,35)$ & $19,40(4,03)$ & 0,81 \\
UOT & $12,33(3,98)$ & $14,60(2,95)$ & $12,47(2,50)$ & 1,74 \\
\hline
\end{tabular}

\section{Discussão}

Considerando-se que crianças com autismo apresentam resultado abaixo do esperado em tarefas que avaliam teoria da mente e que déficit de ToM tem sido considerado um possível endofenótipo para o transtorno (Best et al., 2008), o presente estudo teve como objetivo avaliar a presença de déficit nesta habilidade também em familiares de pessoas com autismo. Em consonância com o estudo de Gokcen et al. (2009) não foi encontrada diferença significativa entre os grupos na habilidade de decodificação da teoria da mente. Por outro lado, houve diferenças expressivas entre os grupos na habilidade de dedução de ToM.

Conforme apontado por Sabbagh (2004), a existência de diferentes redes neurais associadas aos componentes da teoria da mente aponta para a viabilidade de investigação deste constructo separadamente. Os resultados oferecem suporte à noção de que a divisão da teoria da mente entre duas subcategorias da habilidade traz contribuições para o tema em questão. Desta forma, trata-se de uma evidência adicional à apresentada por Gokcen et al. (2009) no sentido da definição de teoria da mente enquanto um conceito multidimensional, divergindo do enfoque de grande parte dos estudos anteriores sobre o tema (Baron-Cohen et al., 2006; Losh \& Piven, 2007; Nýden et al., 2011; Shimoni, Weizman, Yoran, \& Raviv, 2012).
Os achados da presente pesquisa somam-se às crescentes evidências da existência de componentes da teoria da mente que se relacionam a circuitos cerebrais diferentes e não se sobrepõem (Caoa et al., 2012; Njomboro et al., 2008; Sabbagh, 2004; Tager-Flusberg \& Sullivan, 2000). Sugere-se assim que a decodificação do estado mental seria um estágio inicial, enquanto a habilidade de dedução seria a segunda etapa do processo (Njomboro et al., 2008; Sabbagh, 2004), de forma sequencial.

Destaca-se, ainda, a ausência de diferenças significativas entre o grupo de pais de pessoas com síndrome de Down e o grupo de pais de crianças com desenvolvimento típico. Este achado aponta para a noção de que o déficit da habilidade de dedução encontrado em pais de crianças com autismo não se deve ao impacto da presença de uma doença crônica na família.

No que se refere à avaliação em separado de pais $\mathrm{e}$ mães, quanto à teoria da mente, os resultados apontam para a existência de deficits na habilidade de dedução unicamente das mães. Os mesmos resultados comparativos entre pais e mães foram relatados no estudo recente de Shimoni et al. (2012), sugerindo uma contribuição materna mais proeminente na transmissão do endofenótipo da teoria da mente.

Há suporte consistente na literatura sobre a forte herdabilidade do autismo, bem como sobre a presença de FAA 
em parentes de primeiro grau de crianças com autismo (Ronald \& Hoekstra, 2011; Scheeren \& Stauder, 2008). Os resultados do presente estudo, por sua vez, apontam o déficit de ToM, e mais especificamente, os prejuízos na habilidade de dedução, como marcador endofenotípico do autismo, sugerindo ser este um déficit subclínico presente nesta amostra, em consonância com os resultados encontrados por Gokcen et al. (2009) e Shimoni et al. (2012).

Deve-se destacar que os resultados da presente pesquisa não visam a geração de uma explicação causal única para o transtorno do espectro do autismo, uma vez que se trata de um transtorno complexo e multifacetado. Tal apontamento remete à discussão sobre se o autismo (e consequentemente os traços subclínicos parentais) deve ser abordado como um transtorno coerente ou passível de ser compartimentado. Hobson (2014) destaca que uma visão fracionada do transtorno pode estimular intervenções que abordem fragmentos do quadro clínico, enquanto que o ponto de vista da coerência pode levar a um enfoque na remediação de uma via final comum, constituindo-se em uma abordagem mais global. No entanto, uma teoria de déficits múltiplos que aborde variados níveis explicativos para o transtorno e que permita lançar luz a características parentais específicas pode permitir uma priorização das intervenções mais apropriadas tanto para a pessoa com autismo quanto para seus familiares, de modo a alcançar um desenvolvimento global efetivo.

Desta forma, alguns enfoques de pesquisas futuras merecem destaque. Primeiramente, para uma mensuração precisa do construto teoria da mente, no sentido de fornecer mais evidências sobre os possíveis componentes de ToM e a relação entre eles, faz-se necessário o desenvolvimento de instrumentos validados. Além disso, a identificação das diferenças entre pais e mães na manifestação dos diferentes endofenótipos do autismo (teoria da mente, coerência central e função executiva), bem como a investigação sobre as combinações de endofenótipos parentais que contribuem para a emergência do transtorno em sua manifestação clínica, devem ser enfocados em estudos subsequentes, de modo a elucidar os traços parentais que devem ser alvo de atenção, no nível da prevenção, e da intervenção, no tratamento da família com um membro com transtorno do espectro do autismo.

\section{Considerações Finais}

Ao identificar a presença de déficits de habilidade de dedução da teoria da mente na amostra investigada de pais de pessoas com autismo o presente estudo aponta para as seguintes direções: (a) a nível teórico, para a proficuidade da subdivisão da categoria teoria da mente; (b) a nível metodológico, para a importância de estudos que enfoquem a avaliação de pais e mães em separado, bem como para a necessidade de maior padronização dos instrumentos de pesquisa e; (c) a nível técnico, para a necessidade de elaboração de metodologias de intervenção que levem em consideração tais características parentais.

\section{Referências}

Achenbach, T. M., \& Edelbrock, C. S. (1983). Manual for the child behavior checklist/ and revised child behavior profile. Burlington, VT: University of Vermont.

Andrade, A. A., \& Teodoro, M. L. M. (2012). Família e autismo: Uma revisão da literatura. Contextos Clínicos, 5, 133-142. doi:10.4013/ctc. 2012.52 .07

Associação Americana de Psiquiatria. (2002). Manual diagnóstico e estatístico de transtornos mentais (DSM IV) (4. ed.). Porto Alegre, RS: Artes Médicas.

Associação Americana de Psiquiatria. (2014). Manual diagnóstico e estatístico de transtornos mentais: DSM-5 (5. ed.). Porto Alegre, RS: Artmed.

Baron-Cohen, S., Jolliffe, T., Mortimore, C., \& Robertson, M. (1997). Another advanced test of theory of mind: Evidence from very high functioning adults with autism or Asperger Syndrome. Journal of Child Psychology and Psychiatry, 38, 813-822. doi:10.1111/j.1469-7610.1997.tb01599.x

Baron-Cohen, S., Ring, H., Chitnis, X., Wheelwright, S., Gregory, L., Williams, S., ...Bullmore, E. (2006). fMRI of parents of children with Asperger syndrome: A pilot study. Journal of Brain Cognition, 61, 122-130.

Best, C. S., Moffat, V. J., Power, M. J., Owens, D. G., \& Johnstone, E. C. (2008). The boundaries of the cognitive phenotype of autism: Theory of mind, central coherence and ambiguous figure perception in young people with autistic traits. Journal of Autism and Developmental Disorders, 38, 840-847. doi:10.1007/s10803-007-0451-8

Betancur, C. (2011). Etiological heterogeneity in autism spectrum disorders: More than 100 genetic and genomic disorders and still counting. Brain Research, 1380(22), 42-77. doi:10.1016/j. brainres.2010.11.078

Bordin, I. A. S., Mari, J. J., \& Caeiro, M. F. (1995). Validação da versão brasileira do Child Behavior Checklist (CBCL) Inventário de Comportamentos da Infância e da Adolescência: Dados preliminares. Revista ABP-APAL, 17, 55-66.

Caoa, B., Lib, Y., Lia F., \& Lia, H. (2012). Electrophysiological difference between mental state decoding and mental state reasoning. Brain Research, 1464, 53-60. doi:10.1016/j. brainres.2012.05.009

Constantino, J. N. (2011). The quantitative nature of autistic social impairment. Pediatric Research, 69(5 Pt. 2), 55R-62R. doi:10.1203/PDR.0b013e318212ec6e

Dyck, M. J., Ferguson, K., \& Shochet, I. M. (2001). Do autism spectrum disorders differ from each other and from nonspectrum disorders on emotion cognition tests? European Child and Adolescent Psychiatry, 10, 105-116. doi:10.1007/ s007870170033

Folstein, S. E., \& Rutter, M. (1977). Infantile autism: A genetic study of 21 twin pairs. Journal of Child Psychology and Psychiatry, 18, 297-321. doi:10.1111/j.1469-7610.1977. tb00443.x

Fombonne, E. (2009). Epidemiology of pervasive developmental disorders. Pediatric Research, 65, 591-598. doi:10.1203/ PDR.0b013e31819e7203

Gokcen, S., Bora, E., Erermis, S., Kesikci, H., \& Aydin, C. (2009). Theory of mind and verbal working memory deficits in parents of autistic children. Psychiatry Research, 166, 4653. doi:10.1016/j.psychres.2007.11.016

Gottesman, I. I., \& Gould, T. D. (2003). The endophenotype concept in psychiatry: Etymology and strategic intentions. Journal of American Psychiatry, 160, 636-645. 
Andrade, A. A., Camargos Junior, W., Ohno, P. M. \& Teodoro, M. L. M. (2015). Teoria da Mente em Pais de Pessoas com Autismo: Uma Análise Comparativa.

Happé, F., \& Ronald, A. (2010). The 'fractionable autism triad': A review of evidence from behavioural, genetic, cognitive and neural research. Neuropsychological Review, 18, 287-304. doi:10.1007/s11065-008-9076-8

Hobson, R. P. (2014). The coherence of autism. Autism, 18(1), 6-16. doi:10.1177/1362361313497538

Hu, M., Chen, J., Li, L., Zheng, Y., Wang, J., Guo, X., ...Zhao, J. (2011). Semantic fluency and executive functions as candidate endophenotypes for the early diagnosis of schizophrenia in Han Chinese. Neuroscience Letters, 502, 173-177. doi:10.1016/j.neulet.2011.07.037

Jonas, K. G., \& Markon, K. E. (2014). A meta-analytic evaluation of the endophenotype hypothesis: Effects of measurement paradigm in the psychiatric genetics of impulsivity. Journal of Abnormal Psychology, 123(3), 660-675. doi:10.1037/ a0037094

Kanne, S. M., Christ, S. E., \& Reiersen, A. M. (2009). Psychiatric symptoms and psychosocial difficulties in young adults with autistic traits. Journal of Autism and Developmental Disorders, 39, 827-833. doi:10.1007/s10803-008-0688-x

Lichtenstein, P., Carlstrom, E., Rastam, M., Gillberg, C., \& Anckarsater, H. (2010). The genetics of autism spectrum disorders and related neurospychaitric disorders in childhood. American Journal of Psychiatry, 167, 1357-1363. doi:10.1176/appi.ajp.2010.10020223

Losh, M., \& Piven, J. (2007). Social-cognition and the broad autism phenotype: Indentifying genetically meaningful phenotypes. Journal of Child Psychology and Psychiatry, 48(1), 105-112. doi:10.1111/j.1469-7610.2006.01594.x

Mecca, T. P., Bravo, R. B., Velloso, R. de L., Schwartzman, J. S., Brunoni, D., \& Teixeira, M. C. T. V. (2011). Rastreamento de sinais e sintomas de transtornos do espectro do autismo em irmãos. Revista de Psiquiatria do Rio Grande do Sul, 33(2), 116-120. doi:10.1590/S0101-81082011000200009

Nascimento, E. (2004). Adaptação, validação e normatização do WAIS-III para uma amostra brasileira. In D. Wechsler (Ed.), WAIS-III: Manual para administração e avaliação (pp. 161192). São Paulo, SP: Casa do Psicólogo.

Njomboro, P., Deb, S., \& Humphreys, G. W. (2008). Dissociation between decoding and reasoning about mental states in patients with theory of mind reasoning impairments. Journal of Cognitive Neuroscience, 20, 1557-1564. doi:10.1162/ jocn.2008.20118

Nýden, A., Hagberg, B., Goussé, V., \& Rastam, M. (2011). A cognitive endophenotype of autism in families with multiple incidence. Research in Autism Spectrum Disorders, 5, 191200. doi:10.1016/j.rasd.2010.03.010

Pellicano, E., Maybery, M., Durkin, K., \& Maley, A. (2006). Multiple cognitive capabilities/deficits in children with an autism spectrum disorder: "Weak" central coherence and its relationship to theory of mind and executive control. Development and Psychopathology, 18, 77-98. doi:10.1017/ S0954579406060056

Premack, D., \& Woodruff, G. (1978). Does the chimpanzee have a theory of mind? Behavioural and Brain Science, 1, 515-526. doi:10.1017/S0140525X00076512

Rommelse, N. N. J., Geurts, H. M., Franke, B., Buitelaar, J. K., \& Hartman, C. A. (2011). A review on cognitive and brain endophenotypes that may be common in autism spectrum disorder and attention-deficit/hyperactivity disorder and facilitate the search for pleiotropic genes. Neuroscience \& Biobehavioral Reviews, 35(6), 1363-1396. doi:10.1016/j. neubiorev.2011.02.015
Ronald, A., \& Hoekstra, R. A. (2011). Autism spectrum disorders and autistic traits: A decade of new twin studies. American Journal of Medical Genetics Part B: Neuropsychiatric Genetics, 156B, 255-274. doi:10.1002/ajmg.b.31159

Sabbagh, M. A. (2004). Understanding the orbitofrontal contributions to theory of mind reasoning: Implications for autism. Brain and Cognition, 55, 209-219. doi:10.1016/j. bandc.2003.04.002

Scheeren, A., \& Stauder, J. (2008). Broader autism phenotype in parents of autistic children: Reality or myth? Journal of Autism and Developmental Disorder, 38, 276-287. doi:10.1007/ s10803-007-0389-x

Shimoni, H. N., Weizman, A., Yoran, R. H., \& Raviv, A. (2012). Theory of mind, severity of autistic symptoms and parental correlates in children and adolescents with Asperger syndrome. Psychiatry Research, 197, 85-89. doi:10.1016/j. psychres.2012.02.021

Tager-Flusberg, H., \& Sullivan, K. (2000). A componential view of theory of mind: Evidence from Williams syndrome. Cognition, 76(1), 59-89. doi:10.1016/S0010-0277(00)00069-X

Vellante, M., Baron-Cohen, S., Melis, M., Marrone, M., Petretto, D. R., Masala, C., \& Preti, A. (2012). The "Reading the Mind in the Eyes" test: Systematic review of psychometric properties and a validation study in Italy. Cognitive Neuropsychiatry, 18(4), 326-354. doi:10.1080/13546805.2012.721728

Viding, E., \& Blakemore, S. J. (2007). Endophenotype approach to developmental psychopathology: Implications for autism research. Behavior Genetics, 37, 51-60. doi:10.1007/s10519006-9105-4

Wechsler, D. (1981). Wechsler adult inteligence scale revised manual. New York: Psychological Corporation. 